\title{
Preface
}

The world of crickets is a world of scientific adventure and human fascination. Most cricket species are musical, sound-producing insects that have long been part of human life and lore. For instance, according to Polynesian creed, crickets are embodiments of the souls of loved ones, and in many countries a cricket singing at the hearth is thought to bring luck and protect the home against evil spirits. The fierce rivalry behavior of male crickets was well known in ancient China; games were organized and bets were waged on the outcome of their battles. But it is the elaborate behavior of crickets, and especially their acoustic communication, which has always drawn the most attention from biologists and the general public.

This multiauthored book offers an introduction to cricket behavior, especially acoustic behavior, and neurobiology. It tells about the manifold strategies crickets employ in matching development with seasons and habitats, finding mates, and avoiding parasites and predators, and it describes the physiological mechanisms, especially the neuronal mechanisms, underlying cricket behaviors. Even though 64 genera and 116 species of crickets are mentioned, representing the 13 subfamilies of Gryllidae throughout the world, this book is not a monograph; rather it deals with selected aspects of cricket biology assembled under the umbrella of behavior and neurobiology. In fact, most information available from such combined studies comes from fewer than a half dozen species in one subfamily.

Why have crickets remained so interesting to so many generations of students of animal behavior and physiology and become model systems for behavioral physiology and neuroethology? Probably because crickets display elaborate behaviors that can easily be studied in the field and in the laboratory. Some of their behavioral patterns can be measured with high resolution, even in partially restrained animals. These behaviors provide important insights into many sensory, neuromuscular, neuroen- 
docrine, and central nervous system structures and mechanisms at the whole-animal level down to the level of single cells. Furthermore, there are abundant species for comparative studies, several species are readily reared in captivity under seminatural conditions, crickets do not sting, and most do not have painful bites.

We hope this book stimulates cross-fertilization between different biological fields and encourages scientific progress. An evolutionary biologist with a particular interest in natural history, life cycles, geographic distribution, mechanisms of speciation, or species relationships may gain as much from it as might a behavioral ecologist who is trying to understand how different environments influence and shape specific behaviors and to what extent genetic backgrounds or even individual experiences are responsible for optimizing cricket life and reproduction in a given habitat.

Students of comparative behavior and physiology can learn about the various tactics employed by closely and distantly related species. Acoustic cues-important as they may be-are only part of a cricket's communication network. Other sensory stimuli, such as substrate vibration, light, or the use of chemicals, often neglected in the past, may complement the acoustic channel or may play primary roles in such cricket behaviors as the selection of habitats and burrow sites, aggregation and migration, and antiparasitic strategies, to mention only a few. Those interested in the circadian organization of behavior will discover that various activities affecting reproductive success are optimized over time by means of internal clocks located in the nervous system.

Although neuroendocrine and hormonal effects and mechanisms in crickets are less well understood than in other insect groups, some progress has been made in demonstrating the structure and distribution of neuroendocrine cells in the brain and in showing how certain hormones affect behavior. Distinct hormonal titers can now be measured in conjunction with particular behavioral states and motor patterns, offering new approaches to and understanding of sexual receptivity, the functioning of the reproductive system, hormonal feedback, and the interaction between hormonal and nervous systems throughout a cricket's life.

The book summarizes what is currently known about a cricket's visually guided behavior and vision, taking into account structural and functional features of the compound eyes and the visual pathway. Visual cues are used to localize burrows and shelters and, with the help of landmarks, to orient the animal. Photoreceptor cells specialized for polarized light perception have been found in the dorsal rim area, and new information has been gained about color vision in crickets.

Chemoreception in crickets is a rather neglected field of research, although numerous chemoreceptors are present on the surfaces of palps, antennae, and other body parts. Knowing more about their structure and function will help us understand how crickets use them to select certain habitats, food plants, and even sexual partners. 
Vibration-sensitive structures outside the auditory system function to a surprising extent. Crickets can detect disturbances produced by predators approaching on the ground or in flight over a considerable range. A digger wasp-cricket interaction is analyzed in some detail in chapter 7 . Crickets also use self-generated vibrations and air particle movements as well as movement-dependent mechanosensory input as feedback devices to stabilize motor patterns and make them more efficient.

Many mechanoreceptors occur over and within the cricket's body. The book discusses their role in eliciting grooming, in controlling body position, and in escape behavior, and thereby illustrates how they interplay with other sensory modalities during complex behavioral sequences, even in situations previously thought to be the exclusive domain of acoustics.

Not surprisingly, more than half the book is devoted to acoustic behavior, for which crickets are famous, and it is here that the most significant advances are presented. The biomechanical, neuromuscular, and neuronal events associated with sound production by crickets are quite well understood, as is the integration of sounds into overall cricket behavior. The ontogeny of sound pattern production and its genetic basis in males is beginning to be elucidated, and even though they do not sing, we need to examine critically how similar the nervous machinery is in females. The concept of a central pattern generator is examined in cricket stridulation, as is increasing knowledge about how different sensory systems interact by feedback to adjust sound production and songs to the environment, optimizing their efficiency both in loudness and in pattern structure.

Considerable new insights concerning the structure and the biomechanics of cricket ears are discussed, including the complex processes of sound conduction and cross-body interference and their role in acoustic orientation. How the hearing system is adapted to small body size and to the frequency range in which crickets broadcast is now better known. Frequency tuning and discrimination in the ear are described, but the underlying mechanisms are still not well understood. The auditory pathway from the ear to the prothoracic ganglion and further on to the brain has been studied at the single cell level, and we have discerned how carrier frequencies and song patterns are encoded at the various levels and how behaviorally relevant information is extracted. Some of these findings may have relevance for all hearing animals. Correlations and even causal relationships between a particular auditory neuron and aspects of phonotactic and avoidance behavior are elucidated. In the brain, cells that act in conspecific partner-recognition have been localized and identified, thereby providing insight into the neuronal hardware of innate releasing mechanisms. This progress was achieved because questions resulting from behavioral studies guided the neurophysiological approach and because sophisticated behavioral and neuronal approaches were combined. Certainly the next and most urgent task will be to study the con- 
nections between neurons forming the recognition and localization network and to explore how they drive the corresponding motor output.

There is evidence for parallel and multineuronal processing within the nervous system of crickets. Thus the concepts of hierarchical organization and command neurons are put into question; they may have to be replaced by ideas of complex interactions between parts of the nervous system, including multimodal and multisensory processing.

Recent studies have demonstrated a high degree of plasticity in cricket nervous systems, and this information needs to be related to neuronal development and behavior, beginning at the level of juveniles. The time may even be near when crickets will become models for experiments on simple forms of learning.

Quite a few unexplored avenues remain-for example, causes and mechanisms of aggression and territoriality, of spacing of individuals within a population, and of differences in sympatric life styles. We are just beginning to appreciate the complexity of sound communication in the natural habitat, where the frequency, pattern, and loudness of sound signals are changed by the environment. There is still little understanding of age-dependent changes in cricket behavior, the genetic basis of senderreceiver interactions, and temperature effects in communication. We know little about how different species of crickets choose their specific habitats or display satellite strategies. All these gaps in our knowledge call for more comprehensive combinations of field and laboratory analyses.

Many topics must await a subsequent book. Here we provide a comprehensive treatment of comparative cricket behavior, one composed of contributions from people who have sought to explain some of life's incredible complexity by seeking physiological mechanisms that control or enhance the behaviors they have studied. It is at once a book about communication, comparative physiology and anatomy, and environmental interaction, for some of the studies and all of the behaviors relate to activities in which crickets engage in nature. Untold numbers have enjoyed crickets; relatively few have studied them seriously; and even fewer have stimulated others to search for answers to basic biological and philosophical questions through their publications and their personal magnetism and enthusiasm. It is for all who may come to love the world of crickets that this book is written.

Assembling a book with many authors requires careful selection of contributors who are cooperative and compatible, who have interesting perspectives to offer, who are active in the field, and who are able to detail the present state of cricket research both at conceptual and at experimental levels. We believe we have succeeded beyond our original hopes, and we most sincerely thank our contributors for their patience and their good efforts. We also thank Robb Reavill, Science Editor, Marilyn M. Sale, Managing Editor, Helene C. Maddux, Associate Manuscript Editor, and 
Cynthia Gration, Marketing Manager, Cornell University Press, Lois Smit and Margo Quinto, copy editors, and Mark F. O'Brien, Theresa Duda, John B. Klausmeyer, Celia O. Riecker, and Margaret Van Bolt, University of Michigan, for their assistance, enthusiasm, and care in bringing this book to completion.

Franz Huber

Thomas E. Moore

WERNER LOHER

Seewiesen, Federal Republic of Germany

Ann Arbor, Michigan

Berkeley, California 



\section{CRICKET BEHAVIOR}

AND NEUROBIOLOGY 
A cricket is

a lovely thing

that likes to sing.

Rose Ann Walker, age 9 (Kevan 1974)

Hark to those tinkling tones,- the chant of the suzumushi!

- If a jewel of dew could sing, it would tinkle with such a voice!

Hoshu Tokunaga, 1871 (Hearn 1898) 